\title{
Computed Laminography of CFRP using an X-ray Cone Beam and Robotic Sample Manipulator Systems
}

\author{
Charles E. Wood ${ }^{\mathrm{a}}$, Neil S. O'Brien ${ }^{\mathrm{b}}$, Andriy Denysov ${ }^{\mathrm{c}}$, Thomas Blumensath ${ }^{\mathrm{a}, *}$ \\ ${ }^{a}$ Signal Processing and Control Group, University of Southampton, UK \\ ${ }^{b} \mu$-VIS X-ray Imaging Centre, University of Southampton, UK \\ ${ }^{c}$ Nikon Metrology, Tring, UK
}

\begin{abstract}
Carbon fibre reinforced polymers (CFRPs) are of interest to the aerospace sector for meeting future $\mathrm{CO} 2$ emission targets due to their weight reduction potential. However, the detection of structural and matrix defects is crucial for determining the performance and suitability of CFRPs in current and future generations of aircraft. Computed laminography (CL), a well-established nondestructive testing method, is well-suited to the scanning of CFRP components with large aspect ratios, for which conventional computed tomography is less suitable. Utilising an existing Nikon Metrology custom build X-ray CT scanner, two lift-in lift-out robotic sample manipulator systems are used to extend the capability of the system and allow the exploration of atypical scanning geometries. Implementing raster and limited angle trajectories, reconstructions using the ASTRA Tomography Toolbox and the SIRT algorithm are able to show structural defects in CFRPs, despite the reduced information inherent with CL systems. This paper reports on the system design and initial experiments that demonstrate benefits and drawbacks of different design options and scanning trajectory choices.
\end{abstract}

Keywords: Computed laminography, hexapod, robot arm, CFRP, X-ray cone-beam.

*Corresponding author: thomas.blumensath@soton.ac.uk 


\section{Introduction}

In 2009, the International Civil Aviation Organization (ICAO) announced a cap on aviation CO2 emissions, starting in 2020, to provide carbon-neutral growth, and a $50 \%$ reduction in $\mathrm{CO} 2$ emissions by 2050 relative to 2005 levels. Furthermore, the ICAO seeks an average 1.5\% improvement in fuel efficiency every year between 2009 and 2020 [1]. To meet this demand, materials that have a high weight reduction potential are of interest to the aerospace sector for improving the fuel efficiency of current and future generations of composite aircraft. For this reason, carbon fibre reinforced polymers (CFRPs) are becoming increasingly popular, despite their high manufacturing cost. The safe and efficient use of novel CFRP components in the aerospace sector requires the availability of non-destructive testing procedures, which are vital both during manufacture to ensure defect free and dimensionally accurate components as well as during aircraft maintenance to detect and monitor potential damage.

Ultrasound and thermographic methods are commonly employed for matrix defect detection in CFRPs [2], with X-ray computed tomography (CT) commonly used to detect structural defects, such as distortions and wrinkles,

however, X-ray CT can also be used to detect impact delamination, inclusions and porosity [3]. The problem for CT arises when there is a requirement to investigate CFRPs with large aspect ratios, due to geometric constraints and high attenuation in longitudinal directions. The stacking of multiple smaller CFRP samples together can be useful for improving the aspect ratio [4], but this does not solve the problem with scanning larger CFRP samples. Furthermore, modern aerospace components are not flat but often have complex shapes making stacking difficult. Dual-energy scanning methods can improve reconstruction results by optimising the two different energy levels for the long and short path lengths through a CFRP sample [5], and have been shown to improve both signal-to-noise ratio (SNR) and contrast-to-noise ratio (CNR). However, this requires additional scanning time, an important metric for industrial NDT applications. 
Computed laminography (CL) is an approach that does not require a full rotation to acquire useful information for subsequent reconstruction, thus removing the geometric constraint for larger CFRP samples. Scan times using CL can also be reduced, since it typically uses relatively few radiographs acquired with a single peak energy, though dual-energy scanning could also be applied to CL. In the context of CFRP, a combined approach using synchrotron-radiation computed laminography (SRCL) and synchrotron-radiation computed tomography (SRCT) has been used to capture 3D images of damage mechanisms [6]. Structural defects within CFRPs have also been investigated using only SRCL [7]. For an extensive literature review on the use of laminography at synchrotron see [8].

Commercial lab based laminography systems are currently available, mainly for circuit board inspection $[9,10]$. Bespoke lab based systems have also been used for laminography. There is a wide range of motions that have been suggested including circular motions $[11,12,13]$ and [14] as well as linear motions [15]. An extensive reference list to previous approaches can be found in [5].

To allow the investigation of a wide range of potential motions, two laboratoryscale systems were designed to convert a Nikon Metrology custom X-ray CT scanner into a CL system. The design and implementation of a custom lift-inlift-out (LILO) hexapod sample manipulator system, and a custom LILO sixaxis robot arm, will be detailed. This will be followed by a comparison between radiography, raster and limited angle CL scan trajectories, and conventional CT. We aim to show that CL is capable of detecting defects within a CFRP sample, achieving scan times that are appropriate for industrial applications.

\section{Materials and Methods}

The Nikon Metrology custom X-ray CT scanner is located within the $\mu$-VIS $\mathrm{X}$-ray Imaging Centre at the University of Southampton. It has a temperaturecontrolled walk-in bay, containing two micro-focus X-ray sources: $225 \mathrm{kVp}$ and $450 \mathrm{kVp}$, and two X-ray detectors: a 16-inch, flat panel with high sensitivity 
CsI scintillator and $200 \mu \mathrm{m}$ pixel pitch (Perkin Elmer XRD 1621 CN3-HS) and a curved linear detector array (CLDA). For the experiments reported here, we used the $225 \mathrm{kV}$ micro focus $\mathrm{x}$-ray source with a rotating anode tungsten reflection target with flat panel detector. Samples up to $100 \mathrm{~kg}$ in weight with a maximum volume of $1 \times 1 \times 1.5 \mathrm{~m}$ can be accommodated. A large user labyrinth towards the rear of the bay permits access for cables and pipes that can be used for custom rigs.

\subsection{General design considerations}

The systems were designed to allow a range of geometric magnifications with a desired range from two to ten. Sample weights of up to 5 kilograms were to be accommodated. Sample size is restricted by the space in our scanner, which can easily accommodate samples of over one meter cube. The systems were designed for region of interest scanning with the requirement that each point in a region of interest can be moved across the entire field of view at a given magnification. To simplify the integration of the control systems of the scanner and the new sample manipulators, it was decided to use the existing scanner motions (such as source, detector and sample stage motions), only for initial alignment of source, sample and detector. During scanning, all motions were anticipated to be performed with the new sample manipulators. This means that source to detector distance remains fixed during scanning.

\subsection{Custom Platform}

The scanner is a carefully calibrated machine, which is used regularly for a wide range of projects. To avoid the custom CL rig interfering with normal scanner operation, the decision was made to bypass the existing sample mounting plate. Using existing 2D drawings and manufacturer part numbers, a 3D CAD model of the scanner was developed using SolidWorks (figure 1). This was subsequently used to design a custom platform that would fit the linear rails and surround the existing sample manipulator rotate stage. The custom platform was designed to be manufactured entirely of aluminium, keeping in mind the 
100kg loading limit of the scanner. An FEA analysis in SolidWorks was performed to ensure that the custom platform could support each CL system (max deflection was below 30 micrometer under static loading with the manipulator and sample.). The platform was also designed with the ability to be driven by the existing sample mounting system, a feature that enables a broad positioning of both CL systems within the X-ray cone beam. Thrust screws are used to reduce backlash between the custom platform and the existing manipulator stage.

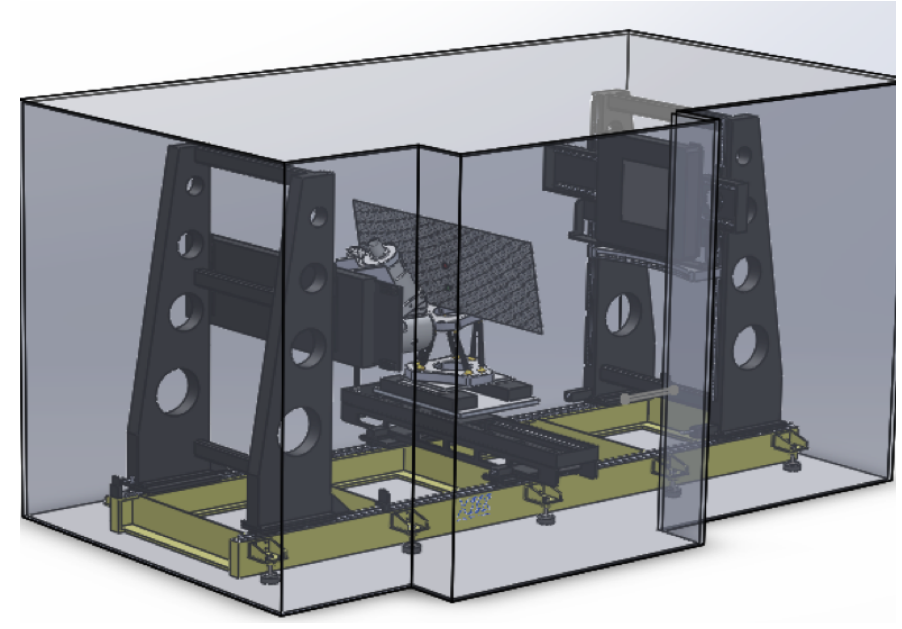

Figure 1: An interactive, programmable 3D model of the custom-built X-ray scanner within the $\mu$-VIS X-ray Imaging Centre at the University of Southampton, showing transparent boundary of X-ray bay.

\subsection{Hexapod Sample Manipulator System}

A hexapod is comprised of a bottom plate and a top plate, connected by six linear actuators, which together offer six DOF and the ability to explore various scanning trajectories. A Physik Instrumente (PI) H-900K013 extended range hexapod was chosen for our application. The working envelope of a hexapod is limited inherently by its design. To extend this the PI hexapod is mounted to a PI LS-270 linear stage to provide additional translational movement. The 
rotation of the hexapod top plate relative to its bottom plate is limited by the travel of the actuators. An additional PI PRS-110 rotation stage is mounted to the top of the hexapod to provide full rotation, which allows our CL system to also perform CT (figure 2). The system is controlled using a PI C887.52 hexapod controller, and is sufficient to support large CFRP specimens.
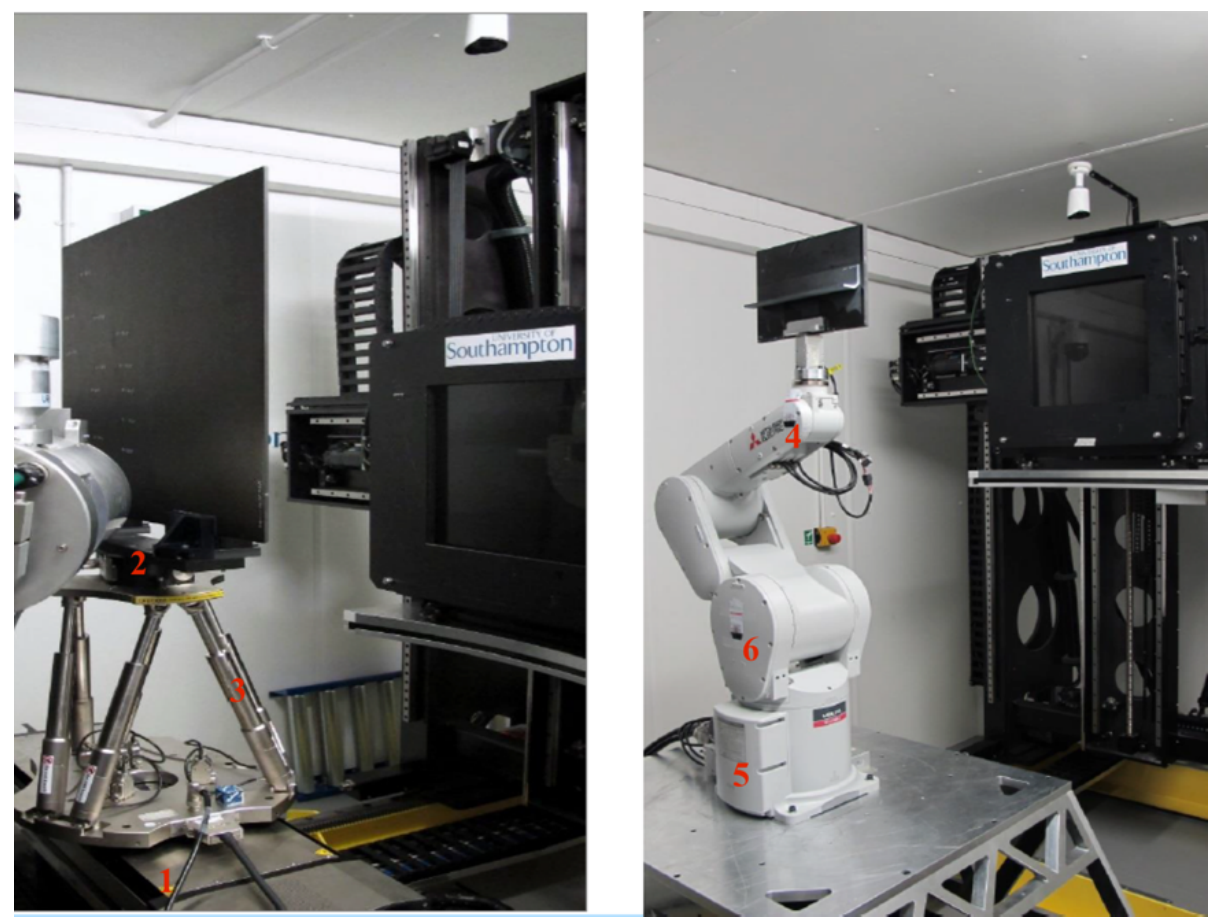

Figure 2: Lift-in-lift-out systems for computed laminography, shown in-situ within the custom-built X-ray CT scanner. Hexapod system shown on the left and robot arm system on the right. Red numbers mark the locations of the $\mathrm{x}$-ray dosimeter mounting locations.

At a magnification of 2 , the hight and width of our detector $(400 \mathrm{~mm}$ by $400 \mathrm{~mm}$ ) specifies a field of view in each radiograph that spans a $200 \mathrm{~mm}$ by 200 $\mathrm{mm}$ region. We set this area as the maximal desired region of interest (RoI) size within the $\mathrm{x}-\mathrm{y}$ plane of a sample. For our desired linear trajectories, to utilise the maximum cone angle at each point within this RoI, a linear travel 
of $200 \mathrm{~mm}$ in the (horizontal) $\mathrm{x}$-axis would be required. This would allow any feature within the RoI to be moved horizontally across the detector for any geometric magnification setting more than 2 . In this way, each point in the RoI could be moved through the X-ray cone to utilise the largest range of X-ray beam angles. We also specified a $40 \mathrm{~mm}$ travel in the (vertical) $\mathrm{y}$-axis. The horizontal movement can be achieved using the linear axis together with the hexapod movement whilst the horizontal movement is achievable using the hexapod alone. The rotation stage furthermore allows the rotation around an axis of rotation that can be angled relative to the source to detector axis using the hexapod.

A custom sample holder was designed and manufactured from Delrin, which does not introduce significant scatter (in contrast to metal parts), and has a low x-ray attenuation coefficient. Several inserts exist to accommodate CFRP samples of different thicknesses. To accommodate large CFRP samples two Delrin sample holders can be used in conjunction with an extension plate.

The relevant specifications for the hexapod are given in table 1, whilst the specifications for the linear stage and rotation stage are given in table 2 . To shield the encoder of the PRS-110 rotation stage from the main X-ray cone beam, lead is applied. Dosimeters are placed on the rotation stage, at the base of the hexapod, near its encoders, and on the LS-270 linear stage, near the its encoder.

\subsection{Six-Axis Robot Sample Manipulator System}

A Mitsubishi RV-7F six-axis robot arm, with an R56 teach box and CR750D controller, was chosen based on its specified repeatability and accuracy. It possesses a larger working volume than the hexapod CL system and can support

large CFRP specimens. An optional I/O card for the CR750-D controller was used in conjunction with a level shifter, an Arduino Mega 2560 and an external 24VDC power supply to communicate with the Nikon Inspect-X scanner control software for data acquisition. An interface plate was manufactured to attach the Delrin sample holder to the robot. The relevant specifications for the robot arm 


\begin{tabular}{|l|l|}
\hline Positional accuracy $(\mu \mathrm{m})$ & \pm 20 \\
\hline Positional accuracy $(\mathrm{deg})$ & \pm 0.003 \\
\hline Uni-directional repeatability $(\mu \mathrm{m})$ & $<0.5$ \\
\hline Uni-directional repeatability $(\mathrm{deg})$ & $<0.001$ \\
\hline Max. load capacity $(\mathrm{kg})$ & 12 \\
\hline Travel range (mm) & $\pm 120, \pm 120, \pm 60$ (x,y,z - single axis) \\
& $\pm 1, \pm 40, \pm 40(\mathrm{x}, \mathrm{y}, \mathrm{z}-$ compound axes $)$ \\
\hline & $\pm 15, \pm 15, \pm 15(\mathrm{Rx}, \mathrm{Ry}, \mathrm{Rz}$ - single axis $)$ \\
Travel range (deg) & $\pm 7.5, \pm 7.5, \pm 7.5(\mathrm{Rx}, \mathrm{Ry}, \mathrm{Rz}$ - compound \\
& axes $)$ \\
\hline Weight $(\mathrm{kg})$ & 22 \\
\hline
\end{tabular}

Table 1: Summary of mechanical specifications of hexapod

\begin{tabular}{|l|l|l|}
\hline Component & LS-270 Linear Stage & $\begin{array}{l}\text { PRS-110 Rotation } \\
\text { Stage }\end{array}$ \\
\hline Positional accuracy $(\mu \mathrm{m})$ & \pm 3 & N/A \\
\hline Positional accuracy $(\mathrm{deg})$ & N/A & 0.01 \\
\hline Uni-directional repeatability $(\mu \mathrm{m})$ & 0.5 & N/A \\
\hline Uni-directional repeatability $(\mathrm{deg})$ & N/A & 0.002 \\
\hline Max. load capacity $(\mathrm{kg})$ & 150 & 10 \\
\hline Travel range $(\mathrm{mm})$ & 300 & $\mathrm{~N} / \mathrm{A}$ \\
\hline Travel range $(\mathrm{deg})$ & $\mathrm{N} / \mathrm{A}$ & 360 \\
\hline Weight $(\mathrm{kg})$ & 29 & 2.6 \\
\hline
\end{tabular}

Table 2: Summary of mechanical specifications of linear and rotation stages 


\begin{tabular}{|l|l|}
\hline Max. load capacity $(\mathrm{kg})$ & 7 \\
\hline Weight $(\mathrm{kg})$ & 65 \\
\hline Maximum reach radiusy $(\mathrm{mm})$ & 713 \\
\hline Positional accuracy $(\mathrm{deg})$ & \pm 0.003 \\
\hline Repeatability to ISO 9283 $(\mathrm{mm})$ & \pm 0.01 \\
\hline Absolute accuracy $(\mathrm{mm})$ & 0.2 \\
\hline
\end{tabular}

Table 3: Summary of mechanical specifications of Mitsubishi RV-7F robot arm

are given in table reftable3. Dosimeters are applied near the specimen holder, at the base of the robot arm, and on joint 2 (see figure 2).

\subsection{Communication interfaces}

The $\mu$-VIS X-ray Imaging Centre currently uses Nikon Metrologys Inspect-X version 2. This enables macros to be written in Visual Basic for Applications (VBA) and subsequently run for custom applications. A USB interface is also present for interfacing hardware with the acquisition computer. Custom VBA macros for Inspect-X were written which communicated with the PI hexapod controller using a serial interface (via an FTDI USB to RS-232 converter). The macros generate the coordinates of each point in the trajectory and request the controller move to the point. Once the hexapod system has moved the sample to a commanded position, and come to rest, a radiograph is acquired and metadata written, including the commanded and reported positions, as well as X-ray beam and imaging system parameters. This is repeated until the required number of projections has been obtained. The I/O card of the Mitsubishi CR750-D controller for the six axis robot arm provides 32 digital inputs and 32 digital outputs which can be used to control the robot. Using a purpose built interface circuit driven by an Arduino micro-controller we were able to interface the X-ray system control software with the robot arm. The interface allowed us to request the robot to move to the next commanded scan 
position at which point the robot returns a signal that it had reached the desired location. However, this communication interface does not allow us to also record the actual positions of the robot as reported by the internal robot encoders. A proprietary software package by the robot arm manufacturer enabled robot programmes that generate the actual coordinates of the scan trajectory to be written in a version of BASIC and simulated before copying the programme to the controller for live robot operations.

\subsection{Scan trajectories}

Both manipulator systems allow a wide range of scan trajectories to be used. We here limited the study to two trajectories, raster and limited angle. The raster scan trajectory moved the sample to positions on a regular 12 x 12 grid of points in the plane orthogonal to the x-ray beam. The limited angle scans used different amounts of rotation around a fixed axis. For both scan trajectories 144 projections are acquired unless noted otherwise. Raster scans using a stationary source and detector have been investigated for CL previously [11] whilst limited angle scanning for CL can use and adapt many of the reconstruction algorithms for CT since it represents a subset of full CT [5]. For this initial investigation of CL these scanning trajectories therefore serve as a reasonable basis from which to proceed and perform relatively short scans, including RoI scans, for CFRPs of various sizes. For the six axis robot arm, a standard pallet routine was adapted to perform a raster motion, allowing easy definition of the size of the rectilinear grid, the step size, and the direction of travel.

\section{Results}

The scanned CFRP specimen was manufactured at the National Composites Centre, with a copper mesh lightning strike protection on one side, and several impacts with a range of energies. The specimen measures 300 x 300 x 5 mm, and weighs approximately $0.7 \mathrm{~kg}$.

Using the raster scan trajectory described in section 2.4 , RoI scans of a moderate energy impact were performed with the hexapod and robot arm system. 
We also performed limited angle scans using the hexapod system using an angular range of 28.5 degrees and of 110 degrees respectively. The 28.5 degrees were selected to mimicking the cone angle used in the raster scans with our system. In the raster scan, the range of angles used at each point depends on the magnification and source-to-detector distance. The 110 degree limited angle scan used 110 projection images. Each scan was accompanied by additional calibration scans. In these scans, we attached high precision $2 \mathrm{~mm}$ ball bearing to the sample and performed several simple motions that allowed us to estimate source, detector and sample positions as well as the main linear and rotation axis of the manipulator. A detailed discussion about the calibration scans and data analysis will be provided in a future publication.

The laminography scans were reconstructed using 20 iterations of the Simultaneous Iterative Reconstruction Technique (SIRT) algorithm as implemented in the ASTRA Toolbox $[16,17]$. Voxels were constrained to be positive. We initialised the SIRT algorithm with a normalised backprojection. The normalised backprojection was computed by first computing a backprojection using all projections. This was normalised by dividing each voxel value in this first backprojection by the voxel value computed by backprojecting virtual projection images where each pixel was set to a value of 1 . This normalisation was useful as different areas in the volume were projected onto different numbers of projection images (areas at the boundary of the RoI contributed to fewer projections than those in the centre of the RoI). Finally, we scaled the normalised backprojection so that the norm of the forward projection of the initial backprojection matched the norm of the observed projection images. The required forward and backward projections were also done using the ASTRA toolbox. ASTRA is a toolbox for tomography that utilises high-performance GPU primitives and which is suitable for 3D X-ray cone beam geometries. ASTRAs SIRT algorithm is well-suited for CL due to the fewer projection angles that are acquired compared to CT. Some of the reconstructions with the raster scan do show horizontal and vertical line artefacts. These are aligned with the motion directions of the scan trajectory and can be suppressed using Discrete Fourier 
Transform based filtering.

For each scan the geometric magnification was approximately 10, the scanned area measured approximately $40 \mathrm{~mm} \times 40 \mathrm{~mm}$ and the accelerating voltage was $90 \mathrm{kV}$. The hexapod raster scan used a current of $215 \mathrm{~A}$ and an analogue gain of $30 \mathrm{~dB}$. The exposure time was $134 \mathrm{~ms}$, whilst the number of frames averaged for each radiograph recorded was 8 . The total scan time was 22 minutes 28 seconds. For the robot arm raster scan a current of $213 \mathrm{~A}$ was used. The analogue gain was $30 \mathrm{~dB}$ and the exposure time was $134 \mathrm{~ms}$. The number of frames averaged was 8 , and the total scan time was 14 minutes and 28 seconds.

To keep a magnification of ten, as well as to achieve the required clearance between the source and the sample, the source to detector distance was increase relative to the one used for the raster scan. This meant that to prevent the reduction of effective X-ray flux, for the hexapod limited angle scans we had to increase the current to $556 \mathrm{~A}$. We set the analogue gain of $25 \mathrm{~dB}$. The exposure time was $354 \mathrm{~ms}$, whilst the number of frames averaged was 4 . The total scan time was then 17 minutes and 45 seconds for the 28.5 degree scan. A summary of the parameters for both raster and limited angle scans is presented in table 4 .

The same RoI was imaged using a region of interest CT scan with 6003 projections, which enabled a comparison to be made between radiography, CL and CT. CT reconstruction was performed using the FDK algorithm using Nikons CT Pro 3D software.

Figures 3 to 6 show some of our results. Figures 3 and 4 show a sub-region of $10 \mathrm{~mm}$ by $10 \mathrm{~mm}$ from a single slice that lies just under the copper mesh, where a high attenuating feature is visible. Figure 3 shows the contrast between the information that can be seen in a single radiograph, in our laminography reconstruction and in a full CT reconstruction. As anticipated, the laminography scan sits in between these extremes in terms of feature resolution and visibility. When looking through the laminography reconstruction slice by slice, there is a clear and strong blurring between nearby slices, which is to be expected in laminography which uses a very limited number of x-ray angles as done here. 


\begin{tabular}{|l|l|l|l|l|}
\hline \multirow{2}{*}{ Scan type } & \multicolumn{2}{|c|}{ Raster scan } & \multicolumn{2}{c|}{ Limited angle hexapod } \\
\cline { 2 - 5 } & Hexapod & Robot & $28.5 \mathrm{deg}$ & $110 \mathrm{deg}$ \\
\hline $\begin{array}{l}\text { Number of projec- } \\
\text { tions }\end{array}$ & 144 & 144 & 144 & 110 \\
\hline $\begin{array}{l}\text { Geometric magnifi- } \\
\text { cation }\end{array}$ & 10 & 10 & 10 & 10 \\
\hline Scanned area $\left(\mathrm{mm}^{2}\right)$ & 40 & 40 & 40 & 40 \\
\hline Voltage $(\mathrm{kV})$ & 90 & 90 & 90 & 90 \\
\hline Current $(\mu \mathrm{A})$ & 215 & 213 & 556 & 556 \\
\hline Gain $(\mathrm{dB})$ & 30 & 30 & 25 & 25 \\
\hline Exposure time (ms) & 134 & 134 & 354 & 354 \\
\hline $\begin{array}{l}\text { Number of frames } \\
\text { averaged }\end{array}$ & 8 & 8 & 4 & 4 \\
\hline $\begin{array}{l}\text { Total scan time } \\
\text { (minutes) }\end{array}$ & 22 & 14 & 18 & 13 \\
\hline
\end{tabular}

Table 4: Summary of raster and limited angle scan parameters 
However, it is also clear that features come in focus in certain slices. Not only does our laminography reconstruction improve the resolution over simple radiography, it also allows us to estimate feature depth. Measuring some of the smallest features in the raster trajectory based laminography reconstruction, we found that the spatial resolution in the plane of the raster scan was about $100 \mu \mathrm{m}$. On the other hand, the resolution in the direction parallel to the plane of the raster motion is not as good as the resolution within this plane and both are worth compared to the full CT results, which, however, require not only a full rotation (which was possible here due to the relatively small sample of CFRP we used) but also used over 40 times more projections.
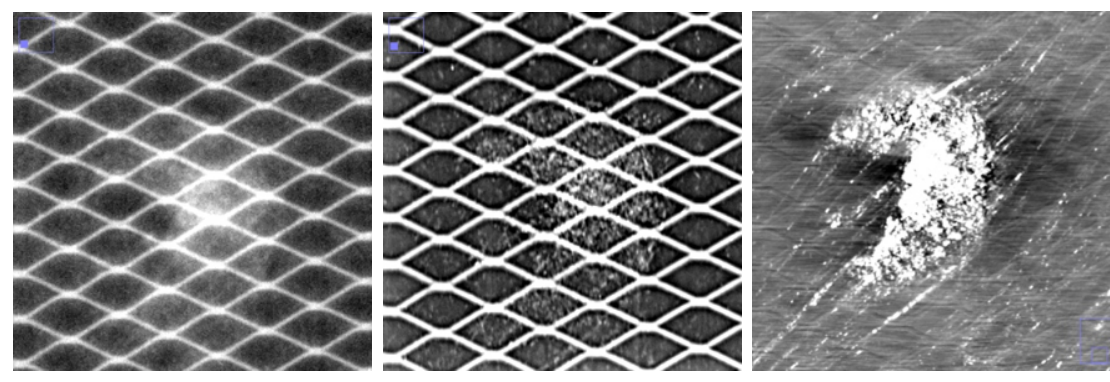

Figure 3: Comparison of a zoomed in view of a radiograph (left), a zoomed in view of one slice from a CL reconstruction (centre) and a zoomed in view of one slice from a CT reconstruction (right). The CL reconstruction was from data acquired with a raster scan trajectory and the hexapod system.

Figure 4 compares the performance of our Hexapod based system with that of the robot arm. It is clear that the Hexapod system leads to a better resolution compared with the robot arm. Reasons for this could be that the repeatability error of the robot arms positional accuracy is larger compared to the Hexapod. Another reason could be that the Hexapod moves along trajectories that are straighter than those of the robot arm. As our reconstruction code assumes the raster scan to be performed along straight trajectories, errors in this will lead to reduced performance. With our communication interface, we were also not able to record the actual (as opposed to the requested) positions of the 

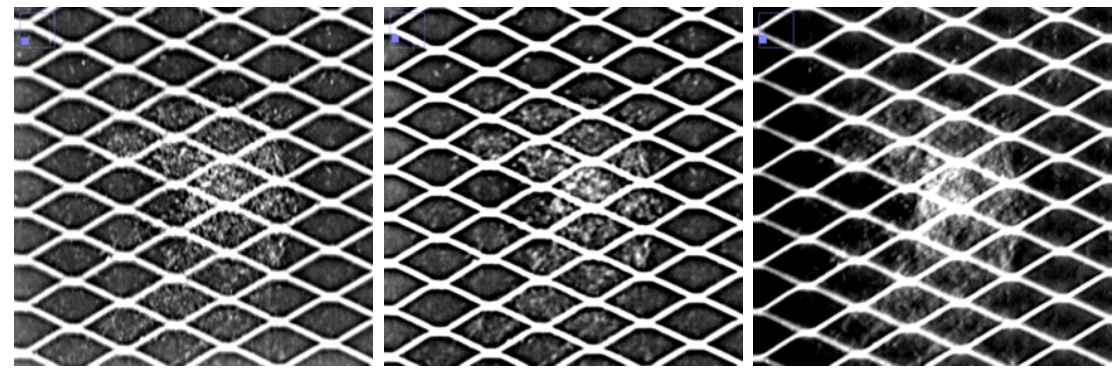

Figure 4: Comparison of a CL reconstruction of a slice towards the front of the panel just underneath the copper mesh using a raster scan trajectory and the hexapod system (left), a CL reconstruction using a raster scan trajectory and the robot arm system (centre) and a CL reconstruction using a limited angle scan (28.5 degrees rotation) and the hexapod system (right). Images are sharper towards the right.
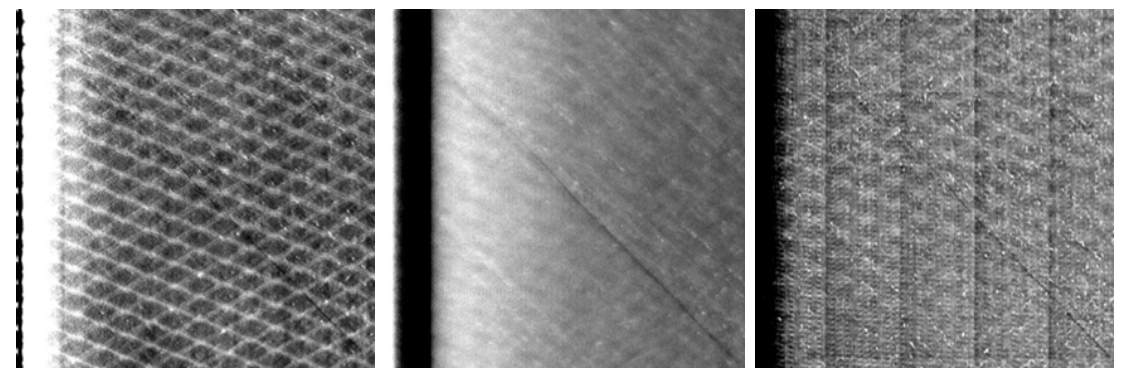

Figure 5: Comparison of a CL reconstruction (zoomed in view) of a slice towards the back of the panel using limited angle scans with 28.5 degrees of rotation (left) 110 degreees of rotation (middle) and a raster scan trajectory (right). Larger imaging angles improves leakage from other layers into the reconstruction. The raster scan however has the sharpest features but also shows some artefacts.

robot joints during scanning. Our reconstruction software could take account of any discrepancies here and this was the approach used for the hexapod system where we had these values. Without these values for the robot arm, a reduced accuracy can be expected. However, when comparing the achieved positions as reported on the robot arm control tool during a scan, these did always seem 

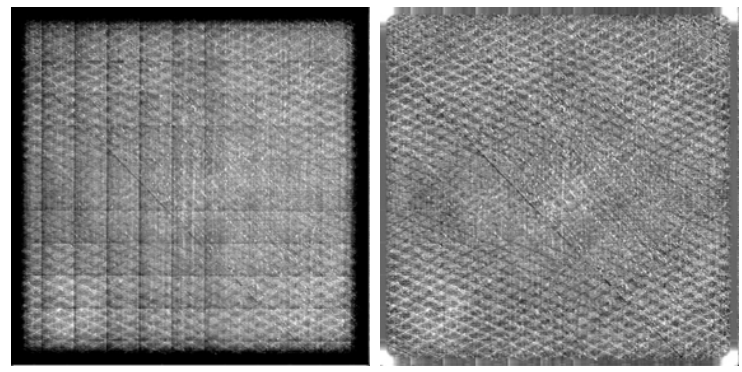

Figure 6: Comparison of a CL reconstruction (full view) of a slice towards the back of the panel using raster scan before (left) and after (right) FFT based artefact reduction.

to exactly correspond to the requested positions. Whilst it is unlikely that the robot arm exactly achieves requested positions, this suggest a limited accuracy either in the positional encoders or in the resolution in the internal position reporting. If this is indeed the case, then recording actual robot arm positions would not improve on the reconstruction results reported here.

Figure 4 also allows us to compare the hexapod based raster scan to the hexapod based limited angle scan. We deliberately tried to match all parameters as closely as possible, including cone angles and the number of projections used. Interestingly, the limited angle reconstruction has a lower resolution than the raster scan reconstruction. One possible reason for this might be that estimating the location and direction of the actual rotation axis relative to source and detector locations is more difficult than estimating the movement axes used for the raster scan and any inaccuracies here will lead to reduced performance.

The limited x-ray angles used here lead to relatively poor resolution in the direction orthogonal to the plane of the raster motion. This is shown for example in Figure 5 where we show a zoomed in portion of another slice of the same reconstruction volume shown in figure 4. In particular, we show two results, those from the limited angle scan (left) and those from the Hexapod based raster scan (right). The reconstruction slice we show exhibits a linear feature with low $\mathrm{X}$-ray absorption. This feature is located near one surface of the sample, on the 
other side from the copper mesh. However, the copper mesh still produces visible artefacts at this layer which is roughly $5 \mathrm{~mm}$ away from the copper mesh layer. This resolution can be improved by using a wider range of X-ray angles. This is shown in the middle of Figure 5, where we show the results from the limited angle scan that used an angular range of 110 degrees. Here the copper mesh is no longer visible. Whilst the resolution orthogonal to the reconstruction plane has been improved, the resolution within the reconstruction plane is, however, still worse than that achieved with the raster scan.

The raster scan on the right of Figure 5 also shows horizontal and vertical line artefacts that were found to be a feature visible in several of our raster scan reconstructions. As stated above, these artefacts can be suppressed using Fourier domain filtering to remove linear and vertical features. The effect of this is shown in Figure 6, where we show the entire slice shown partially in Figure 5. The original reconstruction on the left shows clear linear artefacts. After filtering the image using ImageJs build in FFT based band pass filter option to suppress horizontal and vertical stripes, the artefacts are removed as can be seen on the right in Figure 6.

During many of our scans we did have dosimeters attached to different parts of the systems. The locations are shown in figure 2. Table 5 shows the doses received during our experiments. Whilst it is difficult to compare results across platform (both systems were in the x-ray beam for varying amounts of time and in different amounts of flux), it is clear that the closer we are to the main

beam, the more exposure risk there is. Simple lead shielding however decreased exposure significantly.

\section{Discussion}

These results are extremely encouraging. Even with very few projections, significant detail can be visualised and localised. For example, our results have shown that our laminography scans are able to detect features not visible in simple radiographs. We are also able to locate features in depth, something 


\begin{tabular}{|l|l|}
\hline Location & Radiation dose \\
\hline 1 & $26 \mathrm{mSv}$ \\
\hline 2 & $8 \mathrm{mSv}$ \\
\hline 3 & $35.54 \mathrm{mSv}$ \\
\hline 4 & $159 \mathrm{mSv}$ \\
\hline 5 & $0.4 \mathrm{mSv}$ \\
\hline 6 & $5 \mathrm{mSv}$ \\
\hline
\end{tabular}

Table 5: Radiation dose measured during our experiments on different parts of the manipulator systems. Locations 1, 2 and 3 where on the Hexapod and locations 4,5 and 6 were on the robot arm. The dosimeter at location 2 was under additional lead shielding.

that cannot be achieved by radiographs including inclusions, resin rich areas and even fibre orientation. Of the two trajectories we have compared here, the raster trajectory allows for relatively easy calibration leading to sharper images compared to the limited angle scans. However, the raster scans as implemented have limited resolution orthogonal to the plain of motion. As this resolution is defined by the range of x-ray beam angles used, the raster scan here is restricted to use those angles available in the cone beam angle range.

There are however many advantages to the raster scan trajectory. For example, raster scans can be used to efficiently scan larger areas of a panel whilst limited angle scans can only image a limited region close to the rotation axis.

Both approaches can lead to artefacts. The raster scans often produced vertical and horizontal artefact which could be removed by post-processing, though this could also potentially remove actual vertical and horizontal features. When using the raster scanning techniques, it would thus be advisable to align the scan so that expected linear feature do not align with the scan motion.

The advantages make the raster scan trajectory the preferable approach for the scanning of large flat panel structures, though an approach that increases the 
x-ray beam angle would be desirable. This could be achieved using several raster scans, where in each scan the scan trajectory is at a different angle relative to the plane of the raster motion. For more complexly shaped objects, one can also envisage raster like scan trajectories that do not lie in a plane, but follow more complex surfaces. This should allow the application of the technique to a wider range of aerospace components, such as large composite fan blades found in jet engines. More complex trajectories however would also require more complex calibration.

We were here able to compare two different laminographic systems, one based on a high accuracy hexapod and one based on a six axis robot arm. Due to a $200 \mu \mathrm{m}$ pixel pitch on the detector, the worst acceptable accuracy values in a plane parallel to the detector is defined by the ratio of the pixel pitch and the magnification setting. Thus, using a geometric magnification of 10 for a raster scan trajectory, ideally a system capable of achieving a positional accuracy below $20 \mathrm{~m}$ is required. Our hexapod is nominally able to achieve this, however, common industrial robot arms are not designed for this level of accuracy, which inherently limits the achievable resolution when using this system. However, the robot arm has a much wider working envelope and is also a fraction of the cost of the Hexapod system.

The robot arm we used has other restrictions in the trajectory it uses. The robot arm controller can not currently deal with points that lead to singularities in the positional calculations. Working within the $\mathrm{XZ}$ plane of the robot, this occurred when two joints possess the same x-coordinate value. Furthermore, the movement of the individual robot joints when following a scan trajectory with a sample can lead to configurations where some joints of the robot can easily collide with parts of the scanner. Finding a safe configuration was therefore not trivial, and relied upon the Hutch CAD model and the proprietary software to simulate motions.

Collision risk and subsequent damage remains an important issue when investigating generic scan trajectories. Emergency stops that safely stop the manipulators are thus highly desirable. Whilst our robot system did have such a 
stop, the Hexapod system did not. We thus implemented a software stop, and setting the velocities of stages not used in the current motion to zero, the lack of a hardware e-stop reduced operational control in the event of an emergency. A further potential safety improvement is the inclusion of brakes on the linear actuators of the hexapod. If the hexapod CL system were to lose power, the hexapod would slump. A large sample, constituting an unbalanced load, would then present a danger of damaging X-ray equipment. A final consideration is the possible improvement of shielding. The encoder for the PRS-110 rotation stage is in a position closest to the X-ray cone beam, and therefore has the potential to receive the highest dose. This risk was mitigated with the inclusion of a lead cover, and supported by the readings on the dosimeters that were placed on the CL system. The encoders for the hexapod, located near the base, and the LS-270 linear stage are positioned away from the X-ray cone beam, however, to increase service life lead shielding could be added to these components without impacting their load capacity. The location for the sensor placements is shown below.

There remain a range of important open issues that warrant further investigation. To use iterative reconstruction algorithms, accurate estimates of the source and detector location, detector orientation and reconstruction volume coordinate system are required together with accurate estimates of the position and orientation of the sample manipulator. We here used simple calibration scans in which a small ball bearing was attached to the sample. This allowed an initial estimate of these parameters. More detail on this will be reported in an upcoming publication. Interestingly, errors in the estimation of the source to detector distance do only lead to geometric distortions in the reconstruction and are thus only important if the goal is dimensional measurement. Other parameters such as detector offsets and alignments are however more important for high resolution reconstruction. The accurate estimation of the location and orientation of any rotation axes has been found to be particularly important. Open issues here are a detailed study of the accuracy and repeatability of our manipulator systems and the difference in these between the robot arm and 
hexapod system.

To allow a more principled way to study laminography system performance, more controlled samples are required with well defined features of different orientation and size. As demonstrated here, laminography is very good at detecting sharp features that lie in the main plane of the raster motion or the central plane in the limited angle scan, but features in the direction parallel to this are less well resolved. Test samples need to reflect this and focus on this more difficult direction.

For our initial experiments as reported here, we have kept many of the parameters constant and a more detailed study of the influence of parameters such as the number of projections, signal to noise ratio of each projection and overall scan speed remain to be completed. We have also restricted scanning to two common laminographic scan trajectories and the advantages and disadvantages of more complex trajectories remain to be evaluated.

Results here have been computed using the SIRT algorithm. This choice was made based on initial simulation results that have shown SIRT to generally perform better for laminography than ART, however, a more detailed omparision between different methods on real data as well as an evaluation of different algorithm parameters (such as the number of iterations) remains to be done. The positivity constraint used here was not found to lead to a significant improvement in quality though as it did not significantly slow down reconstruction, it seems advisable to use it. In future, more complex constraints, such as Total Variation constraints might be evaluated, though it is unlikely that total variation constraints are able to significantly improve resolution in the direction orthogonal to the raster scan trajectory given that laminography simply does not collect enough data to provide useful information in this direction. However, we are just starting a project to look at other prior information to overcome this limitation. 


\section{Conclusions}

We have implemented two manipulator systems that allow the study of a wide range of laminographic scan trajectories. This is in contrast to previous work that has concentrated on manipulators with often limited (though possibly more accurate) motion stages. For this first exploration of computed laminography with our systems, we have thus concentrated on the comparison between raster and limited angle scanning trajectories. The focus here was on the detection of features and defects within a CFRP sample, though other applications have been explored. Computed laminography is an attractive alternative to conventional computed tomography for scanning of CFRP specimens with extended aspect ratios, due not only to its ability to mitigate the geometric constraints of most laboratory-scale X-ray CT scanners, but also to overcome the issues of CT when imaging CFRPs along longitudinal axes. In terms of raw data, CL will inherently provide less information than a full CT scan. Thus, CL sits between radiography and full CT in terms of quality. However, we have shown that enough information can be acquired to detect certain structural and matrix defects within CFRP. This research, therefore, serves as a platform for further work, exploring different scanning and reconstruction strategies to optimise speed and exposure time, which will be crucial for the aerospace industry and the widespread adoption of CL for defect detection in CFRPs.

\section{Acknowledgement}

This research was funded by Innovate UK, the UKs innovation agency through ProjectCAN, TSB grant 101804. The ProjectCAN consortium includes QinetiQ, University College London, University of Southampton, Nikon, Axi-Tek and Rolls Royce. 


\section{References}

\section{References}

[1] Price Waterhouse Cooper. Aerospace and defence sector climate change responses [online] (2010). http://www.pwc.co.uk/assets/pdf/climate-changein-aerospace-defence.pdf, Accessed: 2017-04-15.

[2] C. Yang, A. Aneke, M. Gresil, S. O. Oyadiji, Applications of thermography and ultrasonics for detection of debonding in carbon fibre reinforced composite panels, in: 5th International Workshop on Aerostructures, Manchester, 2015.

[3] X. Liu, F. Chen, Defects characterization in CFRP using x-ray computed tomography, Polymers \& Polymer Composites 24 (2) (2016) 149-154.

[4] J. Rouse, Characterisation of impact damage in carbon fibre reinforced plastics by $3 \mathrm{~d}$ x-ray tomography, Ph.D. thesis, The University of Manchester, Manchester (2016).

[5] N. O'Brian, R. Boardman, I. Sinclair, T. Blumensath, Recent advances in x-ray cone-beam computed laminography, Journal of X-ray science and Technology 24 (5) (2016) 691-707.

[6] D. Bull, I. Sinclair, S. Spearing, L. Helfen, Composite laminate impact damage assessment by high resolution 3d x-ray tomography and laminography, in: 8th International Conference on Composite Materials, ICCM 18, 1 Jeju Island, Korea, 2011.

[7] F. Xu, L. Helfen, A. Moffat, G. Johnson, I. Sinclair, T. Baumbach, Synchrotron radiation computed laminography for polymer composite failure studies, Journal of Synchrotron Radiation 17 (2) (2010) 222-226.

[8] L. Helfen, F. Xu, H. Suhonen, P. Cloetens, T. Baumbach, Laminographic imaging using synchrotron radiation challenges and opportunities, Journal of Physics: Conference Series 425 (19) (2013) 1920-1925. 
[9] MatriX Technologies GmbH. Matrix xt-6 [online]. www.nordsonmatrix.com/product/XT-6, Accessed: 2017-04-14.

[10] Nikon metrology. Nikon xt-v series [online]. $\quad$ http://www.nikon.com/products/industrialmetrology/support/download/brochures/pdf/XTV_SERIES_en.pdf, Accessed: 2017-04-14.

[11] M. Rehak, U. Hassler, R. Hanke, Acquisition trajectories for x-ray tomosynthesis applied to planar samples, in: 2nd International Symposium on NDT in Aerospace, Singapore, 2010.

[12] M. Maisl, F. Porsch, C. Schorr, Computed laminography for x-ray inspection of lightweight constructions, in: International Symposium on NDT in Aerospace, Singapore, 2010.

[13] Q. Jie-Min, C. Da-Quan, Z. Wei, T. Xiao, S. Cui-Li, W. Y.-. Fang, W. CunFeng, S. Rong-Jian, W. Long, Y. Zhong-Qiang, Y. Yong-Lian, Computed laminography and reconstruction algorithm, Chinese Physics C 36 (8) (2012) 777-783.

[14] M. Kurfis, G. Streckenbach, Digital laminography and computed tomography with $600 \mathrm{kV}$ for aerospace applications, in: International symposium on NDT in Aerospace, Berlin, 2012.

[15] S. Gondrom, J. Zhou, M. Maisl, H. Reiter, M. Krning, W. Arnold, Xray computed laminography: an approach of computed tomography for applications with limited access, Nuclear Engineering and Design 190 (1-2) (1999) 141-147.

[16] W. van Aarle, W. J. Palenstijn, J. Cant, E. Janssens, F. Bleichrodt, A. Dabravolski, J. D. Beenhouwer, K. J. Batenburg, J. Sijbers, Fast and flexible x-ray tomography using the astra toolbox, Optics Express 24 (22) (2016) 25129-25147. 
[17] W. van Aarle, W. J. Palenstijn, J. D. Beenhouwer, T. Altantzis, S. Bals, K. J. Batenburg, J. Sijbers, The astra toolbox: A platform for advanced algorithm development in electron tomography, Ultramicroscopy 157 (2015) 3547 . 